\title{
LAS COMUNIDADES DE SOFTWARE LIBRE DE COSTA RICA
}

\section{FREE SOFTWARE COMMUNITIES OF COSTA RICA ${ }^{1}$}

\author{
Sofía Flores Solórzano*
}

\begin{abstract}
RESUMEN
Se han investigado las comunidades de software libre de Costa Rica entre 2006 y 2008. Mediante la triangulación metodológica de los datos obtenidos a través de una encuesta electrónica, varias entrevistas y la observación participante, se ha analizado su estructura y funcionamiento desde el concepto "comunidad virtual". Son comunidades de conocimiento, compuestas por usuarios estratégicos, quienes practican cierto elitismo y un discurso socrático.
\end{abstract}

PALABRAS CLAVE: COSTA RICA * INFORMÁTICA * COMUNIDAD VIRTUAL * SOFTWARE LIBRE $*$ CÓDIGO ABIERTO $*$ ASPECTOS TÉCNICOS * SOCIALIZACIÓN

\section{ABSTRACT}

The free software communities of Costa Rica have been investigated between 20062008. To understand their structure and functioning we keep a participant observation and conduct an electronic poll and interviews. The results are analyzed under the virtual community concept. They consist of strategic users, with scarce female involvement, ephemeral proposals, a touch of elitism and share a dialogic discourse.

KEY WORDS: COSTA RICA * COMPUTER SCIENCE $*$ VIRTUAL COMMUNITY $*$ FREE SOFTWARE $*$ OPEN CODE $*$ TECHNICAL ASPECTS $*$ SOCIALIZATION

Docente de la Escuela de Estudios Generales, Universidad de Costa Rica.

sofia.flores@ucr.ac.cr
1 Este artículo es una derivación de mi tesis de Maestría "Las comunidades de software libre de Costa Rica”. Universidad de Costa Rica. 2009. 


\section{INTRODUCCIÓN}

En Costa Rica existen varios grupos que de manera voluntaria se dedican a proporcionar asistencia técnica y a promover el uso de GNU/ Linux $^{2}$. Este trabajo trata de aproximarse a esos colectivos que interactúan predominantemente a través de Internet, aunque también efectúan actividades off line, por ejemplo, para realizar conferencias de promoción de software libre, festivales de instalación, como el Festival Latinoamericano de Instalación de Software Libre ${ }^{3}$ (FLISOL) o el Día de la Libertad del Software (Software Freedom Day). Hasta la fecha no se ha publicado ningún estudio en profundidad sobre los usuarios y desarrolladores de GNU/Linux de Costa Rica. Precisamente, uno de los aspectos importantes para comprender la creciente popularidad de GNU/Linux lo constituyen las comunidades, de ahí que estas sean el principal objeto de este estudio y su precedente (Flores, 2009).

Entonces ¿Constituyen los grupos de usuarios de GNU/Linux de Costa Rica verdaderas comunidades virtuales ${ }^{4}$ o son solamente grupos

Nombre con el que se denomina el sistema operativo compuesto por el núcleo o kernel Linux más las herramientas proporcionadas por el proyecto $\mathrm{GNU}$ (GNU es un acrónimo que significa GNU's Not UNIX, es un proyecto que pertenece a la Free Software Foundation cuyo objetivo es desarrollar un sistema operativo completamente libre basado en UNIX. La FSF tiene el copyright de una gran proporción del sistema operativo GNU/Linux así como de una cantidad importante de software libre.

Es el evento de difusión de software libre más grande en Latinoamérica. Se realiza desde el año 2005 y su principal objetivo es promover el uso del software libre, dando a conocer al público en general su filosofía, alcances, avances y desarrollo. En: $<$ http://www.installfest.info>

El término "comunidad", núcleo de la frase nominal "comunidad virtual", viene del término francés communité, de donde lo toma el inglés community, y nos remite a la raíz latina munis 'carga': comunidad > commūnitätem $>$ commū nitās $>$ commūnis $>$ cum + munis (la preposición cum en latín significa 'con', 'conjuntamente'). Asimismo, el adjetivo "virtual" proviene de la voz latina virtūs (virtūs > virtualis > virtual), esta última con el significado de 'valor', 'valentía', técnicos que interactúan ocasionalmente en la red? ¿Cuál es su composición y funcionamiento? ¿Qué papel juegan en el desarrollo y difusión del software libre en Costa Rica?

Para intentar responder esas preguntas se han seguido los planteamientos de Rheingold (2000), Castells (2001), Scolari (2004) y Wilbur (2000). Rheingold (2000) acuña el término "comunidad virtual" para referirse a los grupos que emergen en línea. Para este autor resulta claro el papel determinante de la tecnología de la Comunicación Mediada por Computadora (CMC) en el surgimiento de comunidades virtuales y por ello, considera las comunidades virtuales en términos de grupos sociales mediados por computadora (computer-mediated social groups). Destaca como las personas adaptan la tecnología diseñada para un determinado propósito y la convierten en un modo de resolver sus necesidades de comunicación. Así, distingue entre "red" como un término informal para referirse a las computadoras interconectadas, $y$ "comunidad virtual", en el sentido de congregaciones que emergen de la red cuando un grupo de personas mantienen discusiones públicas por cierto tiempo y de manera continua, intercambiando no sólo información sino sentimientos, hasta formar auténticas relaciones personales en el ciberespacio ${ }^{5}$.

Castells (2001), por su parte, analiza los orígenes de las comunidades virtuales, que se encuentran tanto en los movimientos contraculturales, movimiento hippie y en las formas de vida alternativa de las comunidades rurales de finales de los años sesenta en San Francisco como en la actividad de los hackers. En este contexto, las primeras conferencias en línea nacen en su gran mayoría de la necesidad de realizar el sueño de la comuna después del fracaso de los proyectos alternativos de muchos grupos contraculturales. Scolari (2004) postula que es

'esfuerzo', 'mérito', 'talento', 'virtud', 'perfección moral'. Sin embargo, en informática el sentido moderno con el que se emplea el término "virtual" es el de algo que físicamente no existe, pero que se puede representar por medio de un software. novela de ciencia ficción "Neuromancer". 
posible comprender la comunidad virtual como una metáfora, de las muchas que por cierto hay en la informática y en las interfaces de usuario. Para este autor, la comunidad virtual encarna hipótesis teóricas y expresa al mismo tiempo diferentes concepciones del hombre $y$ de las relaciones intersubjetivas a través de una interfaz técnica y simbólica. En esta misma línea, Wilbur (2000) considera la comunidad virtual como una simulación de la comunidad, pero con una gran dosis de tradición y muy pocas dosis de pérdida.

Para efectos del presente trabajo, se intenta demostrar como los grupos de software libre y código abierto estudiados constituyen auténticas comunidades virtuales, entendiendo la comunidad virtual en un sentido amplio $y$ metafórico, como una forma de organización social que se caracteriza por interacción social, autosuficiencia colectiva, objetivos en común, sentido de pertenencia, compromiso de grupo y reglas específicas. Todo ello de manera independiente de un espacio físico, en contraste con otras formas de organización. Asimismo, se propone una nueva categorización para los grupos de software libre y código abierto: comunidades de conocimiento, debido a que sus miembros comparten lo que Flichy (2003) denomina un marco de referencia socio-tecnológico, que está en permanente construcción y negociación. En el contexto de la sociedad de la información como proyecto político de desarrollo, dicho marco integra varios usos de la tecnología y la información, permitiendo a estas comunidades contribuir activamente en el fomento de una cultura de la información participativa y horizontal.

Por otra parte, como comunidades de conocimiento, estos grupos buscan "la verdad" a través de un diálogo constante. En ese sentido, se demuestra como el diálogo socrático es la forma de discurso predominante. Al respecto, recordemos que en la antigüedad tardía, antes de que se instalara el monologismo cristiano, surgieron los dos géneros que evidencian el dialogismo del lenguaje y la visión carnavalesca de la vida: el diálogo socrático y la menipea.

Para los propósitos de este trabajo, interesa el primero. Como señala Kristeva (1981), el diálogo socrático tiene un origen popular y carnavalesco, y se hallaba muy extendido en la antigüedad aunque sólo nos hayan llegado los diálogos de Platón y Jenofonte. La función del diálogo socrático era registrar las conversaciones de Sócrates con sus discípulos y preservar dichas conversaciones en la memoria. Los diálogos socráticos se caracterizan, pues, por una oposición al monologismo oficial que pretende ser dueño de la verdad. Así, la verdad socrática resulta de las relaciones dialógicas de los hablantes a través de dos procedimientos: la sincrisis - confrontación de diferentes discursos sobre un mismo tema-y la anacrisis — provocación de una palabra por otra palabra.

\section{METODOLOGÍA}

La integración de técnicas cuantitativas y cualitativas es lo que se conoce en Ciencias Sociales como triangulación, término introducido por Denzin (1970) y que se ha convertido desde entonces en un lugar común en la literatura sobre metodología. Para evitar esa generalización, Kelle (2001) propuso tres modelos de triangulación: el modelo de validación, el modelo complementario y el modelo trigonométrico. En este estudio se ha seguido el modelo trigonométrico, en este modelo, la triangulación es entendida como la necesidad de combinar métodos cuantitativos y cualitativos para conseguir obtener una visión total del fenómeno estudiado.

El procedimiento seguido fue el siguiente, en primer lugar, se realizó una encuesta electrónica (método cuantitativo) a una muestra de 59 miembros de las comunidades previamente identificadas. El instrumento utilizado fue un cuestionario de 14 preguntas cerradas y abiertas. En total, completaron la encuesta 59 personas de distintas comunidades. A continuación, se efectuaron 30 entrevistas en profundidad, 15 de manera electrónica y 15 de manera presencial (método cualitativo). Finalmente, durante más de dos años (de enero de 2006 a julio de 2008), se estudiaron los entornos virtuales, donde ocurre la comunicación entre los miembros de los distintos grupos: sitios web, 
listas de correo e IRC Internet Relay Chat. Lo anterior se realizó mediante la técnica de la observación-participante (método cualitativo), que incluyó también la asistencia y participación en actividades off line organizadas por las comunidades: charlas, reuniones $y$ festivales de instalación.

\section{RESULTADOS Y ANÁLISIS}

En el momento de emprender esta investigación se comprobó la existencia en la red de varias listas de correo, foros $y$ otros espacios donde se reúnen usuarios de GNU/Linux mayoritariamente costarricenses: el Grupo de Usuarios de Linux de Costa Rica (GULCR), el Grupo de Usuarios de Debian de Costa Rica, el Grupo de Usuarios de Open Source de San Carlos, el Grupo de Usuarios de Linux y Software Libre de la Universidad de Costa Rica (LINUXECCI), la Comunidad de Software Libre del Instituto Tecnológico de Costa Rica, el Grupo de Usuarios de Ubuntu de Costa Rica, la Comunidad de Software Libre de la Universidad de Costa Rica y la Comunidad Sibu. Además, existe una red de comunidades: La Red Costarricense de Software Libre $^{6}$, que busca coordinar acciones conjuntas $y$ propicia el encuentro entre las diferentes comunidades.

Sin embargo, según los datos obtenidos conversando directamente con los protagonistas, la primera comunidad surgió en la Universidad de Costa Rica en 1998, por iniciativa de Marcelo Magallón, estudiante de Física, Jimmy Mejía, estudiante de Meteorología, Mario Guerra, funcionario del Centro de Informática y Roberto Magaña, profesor de la Escuela de Física. Es conveniente señalar que cuando nace esta primera comunidad, ya existían en otras partes del mundo grupos de usuarios de mucha influencia: The Bay Area Linux User $\left(\mathrm{BALUG}^{7}\right)$ o The Silicon Valley Linux User Group $\left(\mathrm{SVLUG}^{8}\right)$,

6 En: <URL: http://www.softwarelibrecr.org/index. php>

En: <http://www.balug.org>

8

En: <http://www.svlug.org/> siendo el segundo uno de los más antiguos y con el mayor número de usuarios del mundo. Posiblemente la influencia de estos grupos, muchos de los cuales perduran hasta el día de hoy, fue determinante en el surgimiento de la primera comunidad de usuarios de GNU/Linux costarricense: GULCR.

De acuerdo con Marcelo Magallón (2007), en la primera reunión de GULCR, hablaron sobre el nombre que le darían al grupo y decidieron llamarlo como los grupos norteamericanos, barajaron varias posibilidades, como CRLUG, es decir, 'Costa Rica Linux User Group', pero finalmente se decidieron por una traducción al castellano: GULCR 'Grupo de Usuarios de Linux de Costa Rica'. El primer mensaje fue publicado el 21 de agosto de 1998. Desde noviembre del 2001, el grupo se encuentra inscrito como una fundación sin fines de lucro en el Registro Nacional de Costa Rica. Desde su fundación ha sido la comunidad de software libre más activa y numerosa de Costa Rica.

No obstante, al cierre de la investigación, tal y como lo manifestara el propio Marcelo Magallón (2007), y también lo han reiterado otros miembros de GULCR como Mario Guerra (2007), co-fundador de GULCR, el grupo se encuentra en una etapa de transición. Muchos miembros ya no participan como antes $y$ otros han decidido crear grupos nuevos. El propio Magallón ha creado un grupo de usuarios de la distribución Debian, y Leonardo Jiménez, ex-miembro de GULCR, a inicios del 2006 fundó LINUXECCI 'Grupo de Usuarios de Linux y Software Libre de la Escuela de Ciencias de la Computación e Informática de la Universidad de Costa Rica'.

\subsection{PROPUESTAS EFÍMERAS}

Uno de los problemas de los grupos analizados es su dificultad para consolidarse en el tiempo. Muchos de estos espacios virtuales no siempre se caracterizan por una gran continuidad, al contrario, la mayoría tiene una breve duración. Los niveles de participación también son variables, aunque la tendencia general es entre mediana y baja participación en casi todos los espacios. Sólo muy pocos 
grupos consiguen tener la suficiente continuidad y participación como para constituirse en comunidades virtuales, en el sentido en el que emplea dicha noción Rheingold (2000).

Lo anterior, debido a que no es fácil constituirse en una comunidad y tener éxito en la red, prueba de ello es que el 29\% de los encuestados considera que su grupo no es una comunidad. Algunas de las razones dadas por los usuarios que no creen que su grupo sea una comunidad fueron las siguientes:

- Me parece que aunque es un buen lugar para compartir conocimientos sobre Debian, todavía hace falta más participación y actividades (Mario V., Debian-CR).

- Solamente algunos son comunidad, otros son espectadores (Anónimo, GULCR).

-Por que las actividades se dan por temporadas, no siempre hay buena organización (Eduardo, GULCR).

-Ya dejó de ser una comunidad, ha caído en desuso y apatía de parte de los miembros (Marco, GULCR, Debian-CR).

- No ha crecido mucho (Ernesto, Ubuntu-CR).

No cabe la menor duda de que nos encontramos ante un objeto de estudio volátil y en permanente construcción, tal y como apunta Castells (2001: 66), muchas de las propuestas comunitarias que surgen en la red se caracterizan por ser efímeras $y$ discontinuas, $y$ en ese sentido, las comunidades de GNU/Linux costarricense no son la excepción.

Por ejemplo, entre julio del 2006 y julio del 2007 surgieron cuatro grupos nuevos: el Grupo de Usuarios de Software Libre de la Escuela de Ciencias de la Computación e
Informática de la Universidad de Costa Rica ${ }^{9}$ (LINUXECCI), la Comunidad de Software Libre de la Universidad de Costa Rica ${ }^{10}$, la Comunidad de Software Libre del Instituto Tecnológico de Costa Rica y el Grupo de Usuarios de Ubuntu de Costa Rica. De estos grupos sólo LINUXECCI ha conseguido consolidarse, aunque se ignora por cuánto tiempo. Por ello, es importante reiterar que GULCR sigue siendo la comunidad con mayor permanencia y participación, tras más de una década de existencia.

\subsection{COMUNIDADES DE JÓVENES INFORMÁTICOS}

De acuerdo con la encuesta realizada, solo un $12 \%$ estudia a tiempo completo, mientras que un $42 \%$ trabaja, y un $46 \%$ estudia $y$ trabaja. Lo anterior resulta significativo, pues refleja una población económicamente activa, inmersa en el mercado laboral. La mayoría ejercen profesiones relacionadas con la informática, siendo la profesión más frecuente Ingeniería en Computación (Fig. 1).

FIGURA 1

FRECUENCIAS ABSOLUTAS

DE LAS PROFESIONES DE LOS MIEMBROS

DE LAS COMUNIDADES

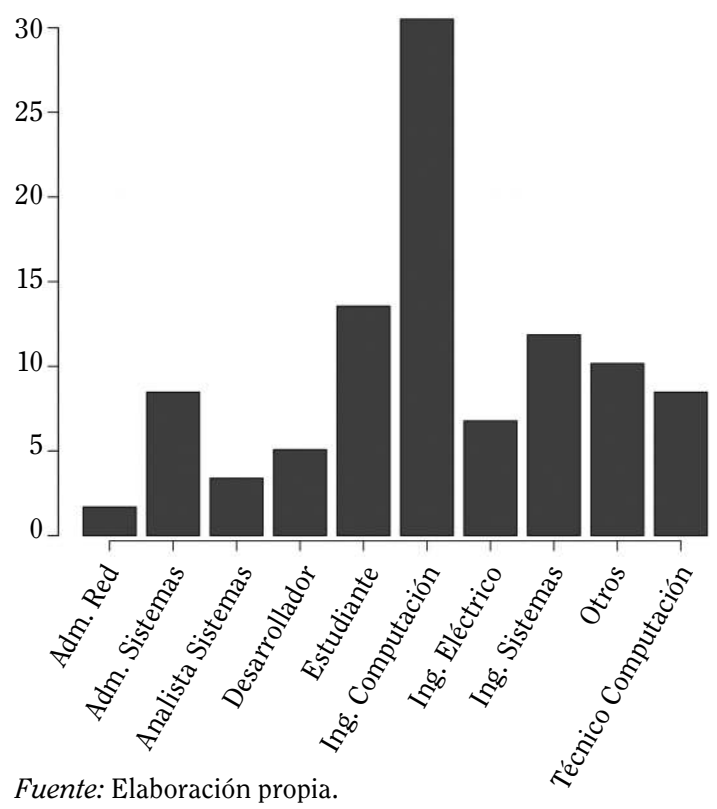

9 En: <http://groups.google.co.cr/group/linuxecci/ about?hl=es>

10 En: <http://softwarelibre.ucr.ac.cr/> 
La mayoría trabaja en el sector privado (66\%), en empresas tan conocidas como Intel, Hewlett Packard o Ericsson de Costa Rica, $y$ en PYMES del sector info-comunicacional, como Gridshield SA, Innovacop, SYSTEC o ITS Infocomunicaciones. Un 10\% labora en alguna institución pública, como el ICE o la Universidad de Costa Rica. El 7\% ofrece servicios profesionales por cuenta propia. Un 5\% no respondió la pregunta $y$ un $12 \%$ corresponde, como se ha comentado anteriormente, al porcentaje que estudia tiempo completo.

\subsubsection{ESCASA PARTICIPACIÓN FEMENINA}

Por otra parte, en todas las comunidades que participaron en la encuesta se constata una escasa presencia de mujeres, el 95\% de la muestra fueron hombres y sólo el 5\% mujeres. En el cuadro 1 podemos ver la distribución más precisa de los miembros por edad y género. Estos datos reflejan lo que de alguna manera es la norma, al menos por el momento, en el mundo del software libre y código abierto: la poca participación de mujeres, tanto en las comunidades como en los proyectos de desarro1lo. Asimismo, en el caso de Costa Rica hay que añadir otro factor, de acuerdo con la percepción de varios estudiantes entrevistados, son pocas las mujeres que deciden estudiar carreras como informática o ingeniería.

\section{CUADRO 1}

DISTRIBUCIÓN DE LOS USUARIOS POR EDAD Y GÉNERO

\begin{tabular}{cccc}
\hline EDAD & HOMBRES & MUJERES & TOTAL \\
\hline $15-20$ & $3 \%$ & $0 \%$ & $3 \%$ \\
$21-26$ & $46 \%$ & $3 \%$ & $49 \%$ \\
$27-32$ & $37 \%$ & $2 \%$ & $39 \%$ \\
$33-38$ & $7 \%$ & $0 \%$ & $7 \%$ \\
$39-44$ & $2 \%$ & $0 \%$ & $2 \%$ \\
\hline TOTAL & $95 \%$ & $5 \%$ & $100 \%$ \\
\hline
\end{tabular}

Fuente: Elaboración propia.

Solamente un $3 \%$ tiene entre 15 y 20 años, es decir, casi no hay adolescentes en estas comunidades. En contraste, la mayoría, un $49 \%$, tiene entre 21 y 26 años, y de este grupo sólo el 3\% son mujeres. Un 39\% tiene entre 27 y 32 años, con un $2 \%$ de mujeres. A partir de los 33 años hay un descenso en la participación, pues solo el 7\% tiene entre 33 y 38 años, $y$ un $2 \%$ tiene entre 39 y 44 años.

Así pues, se concluye que la mayoría de los integrantes de las comunidades de software libre y código abierto de Costa Rica suelen ser jóvenes universitarios o profesionales, en su gran mayoría hombres. Muchos se han iniciado en el sistema GNU/Linux y en el mundo del software libre en la universidad, generalmente a través de compañeros o en algún curso de introducción a la informática, donde además de Unix se comenta algo sobre GNU/Linux.

\subsection{COMUNIDADES DEL CONOCIMIENTO}

Se ha comprobado que no todos los espacios estudiados se ajustan a la definición de comunidad virtual propuesta por Rheingold (2000). La comunidad virtual requiere de un esfuerzo de imaginación y autoconvencimiento, como dice Anderson (1991), basta que exista la idea de comunidad en la mente para que esta exista. Pero parece que no todos los usuarios tienen ese convencimiento ni el compromiso necesario. No cabe la menor duda de que "comunidad virtual" es un término siempre sugerente $y$ atractivo para seguir hablando de espacios como los que se analizan, aunque seamos conscientes de su juego metafórico.

Por todo lo anterior, parece conveniente sustituir la noción de "comunidad virtual" por la de "comunidad de conocimiento". Como comunidades de conocimiento los grupos estudiados presentan características propias. A continuación se expone una selección de los argumentos dados por los miembros que se sienten parte de una comunidad, algunas frases están enfatizadas en letra cursiva, y se brinda el nombre y la comunidad del entrevistado:

-Por el hecho de compartir conocimiento (Moisés, GULCR, Debian-CR, Ubuntu$\mathrm{CR})$. 
- Ayudamos a otros como en una comunidad. Es lo mismo que tener buenos vecinos organizados (José Paulo, GULCR).

- Hay gente especializada en varios campos lista a brindarle una mano a uno (Mauricio, GULCR).

-Existe un sentido de pertenencia; se brinda y se obtiene ayuda regularmente; la comunicación se desarrolla en forma respetuosa y profesional (Marcelo, Desarrollador oficial de Debian, Debian-CR).

- porque comparten conocimiento sin muchos peros (Aurelio, GULCR ).

En la anterior selección de respuestas se evidencia que valores comunitarios como solidaridad, cooperativismo y trabajo colectivo están muy presentes en la concepción que de sí mismos tienen los grupos. Además, como lo señalaba Aurelio en la frase "... porque comparten conocimiento sin muchos peros", la pauta fundamental en este tipo de comunidades es el poder compartir libremente conocimiento. Este conocimiento no es en absoluto abstracto, sino que se refiere a conocimientos técnicos sobre como configurar sistemas GNU/Linux.

El apoyo y la ayuda de la comunidad al usuario es siempre muy importante, pues suele ser el único soporte técnico que existe. Detrás de los proyectos de software libre, generalmente no hay una empresa con un departamento de "ayuda al cliente" al que un nuevo usuario pueda dirigirse en caso de producirse algún fallo. Ese papel lo desempeña la comunidad de usuarios. En ese sentido, la comunidad ha sido el motor principal del software libre, $y$ por supuesto Internet el medio de comunicación que lo ha hecho posible. Sin Internet GNU/Linux no sería lo que es ahora, las comunidades de usuarios de todo el mundo dependen de Internet para poder existir, en palabras de Diego:
- Sí claro, la Internet nos da el ambiente donde nos sentimos juntos y podemos compartir experiencias y soluciones a problemas, todos ayudan a todos (Diego, GULCR, Debian-CR, CCACR, LINUXECCI, UCR).

\subsubsection{USUARIOS ESTRATÉGICOS}

Adicionalmente a lo expuesto, de acuerdo con Flichy (2003), la mayoría de los usuarios no son tácticos, como la mayoría de nosotros, sino estratégicos, es decir, son usuarios que intervienen en el diseño del artefacto tecnológico. $\mathrm{Su}$ papel es crucial, debido a que se encargan de probar las llamadas versiones beta o inestables, envían bugs ${ }^{11}$ a las listas de correo habilitadas para ello y algunos colaboran con tareas concretas como depuración de código y redacción de la documentación.

\subsubsection{ELITISMO Y TECNOMERITOCRACIA}

La mayoría de las comunidades de software libre se caracterizan por la tecnomeritocracia, es decir, los usuarios son valorados por sus conocimientos y capacidad para resolver problemas. Por ello se espera que los usuarios traten de resolver los problemas por sí mismos, leyendo la documentación disponible primero, $y$ luego formulando preguntas concretas $y$ documentadas. Lo anterior ahuyenta a muchos novatos que formulan preguntas consideradas "obvias". De ahí que sea difícil que puedan participar fácilmente si esperan que la comunidad haga todo el trabajo por ellos. Esta actitud no siempre es comprendida, de ahí que para usuarios como Antonio, miembro de LINUXECCI, comunidades como GULCR sean discriminativas y elitistas:

—... Sé que a Mario y a Magallón se les ocurrió hacer un grupo de usuarios de Linux, el problema de GULCR fue que el nivel creció tanto que cuando alguien

Lit. 'bicho'. En la jerga informática un bug es 'un defecto en el código o en la rutina de un programa'. 
nuevo entraba, alguien que no sabía, intentaba unirse al grupo, como que el grupo en sí lo rechazaba. Por eso GULCR está a punto de desaparecer, quedan 3 ó 4. Porque vos hacés una consulta a ese grupo y te tratan malísimo: "lea", "eso es demasiado básico". En el momento en que una comunidad no respete tanto a los que saben mucho como a los que saben poco, tenemos una división. Entonces GULCR se convirtió en un grupo para 4 ó 5 expertos poderosos sabelotodo, que nada más responden si es una pregunta así: "¿Como hacemos para programar en Linux el cohete que está haciendo Franklin Chang Díaz?" entonces sí, todos van a ver lo que es, pero si preguntás "¿Cómo hago para instalar tal cosa?" entonces te van a decir, "no, eso es una estupidez", "sos un idiota". Entonces, nunca más vas a volver a esa comunidad. Tanto así que Mario Guerra ya no está ahí casi, si hay que trabajar en algo Mario Guerra siempre está dispuesto, pero uno lo ve, ellos mismos perdieron el control por permitirlo (...)

En cambio, para la mayoría de miembros de GULCR, el ser considerados elitistas o excluyentes, lejos de ser un defecto, es una virtud, propia de la ética hacker y de las comunidades de software libre, especialmente aquellas en las que participan expertos programadores encargados del desarrollo de Linux. En ese sentido, la cita anterior es reveladora, pues apunta a varios aspectos sobre la configuración de este tipo de tecno-comunidades: tecnomeritocracia, elitismo, poca apertura, severas normas de interacción. En general, son comunidades que tienden a ser cerradas y con un núcleo de gurús al que es muy difícil de acceder si no se hacen los méritos. El tratar de atraer nuevos usuarios siempre ha sido una constante en el mundo del software libre, $y$ las comunidades de Costa Rica no son la excepción. No obstante, por tratarse de grupos muy técnicos, no suelen atraer a todo el mundo, ni crecer mucho, $y$ por eso puede dar la impresión de ser grupos sectarios y cerrados. Por otra parte, prevalece hasta ahora un hackti- vismo digitalmente correcto ${ }^{12}$. La autora de este trabajo ha podido comprobar, en los foros y en algunos festivales de instalación, que predomina una actitud que privilegia lo técnico sobre lo político.

\subsubsection{DIÁLOGO SOCRÁTICO}

Como comunidades de conocimiento, el discurso dialógico está muy presente en las comunidades estudiadas. Las conversaciones de la comunidad son registradas digitalmente y constituyen fuente de información y de consulta, $y$ al leerlas es evidente que estamos ante una forma de discurso ${ }^{13}$.

En nuestra opinión, el modelo del diálogo socrático y los procedimientos retóricos de sincrisis (confrontación de diferentes discursos sobre un mismo tema) y anacrisis (provocación de una palabra por otra palabra) como estructuras discursivas, están presentes en las prácticas de interacción de los grupos en línea, especialmente aquéllos como las comunidades de software libre y código abierto que buscan aclarar dudas sobre tecnología de una manera dialógica y participativa. Este tipo de discurso

Según Lizama Mendoza (2005) se suele dividir el hacktivismo en dos movimientos: El hacktivismo digitalmente correcto, que privilegia lo tecnológico sobre lo político-social, básicamente es el tipo de hacktivismo que realiza una élite de hackers con amplios conocimientos técnicos y el hacktivismo digitalmente incorrecto que privilegia lo político-social sobre lo tecnológico, además de hackers participan activistas sin demasiados conocimientos técnicos.

13 De acuerdo con Kristeva es posible distinguir dos tipos de discursos: el discurso monológico y el discurso dialógico. En el discurso monológico, el principio de organización textual se limita al punto de vista absoluto del narrador que coincide con "el todo de un dios o de una comunidad" (1981: 207). Dentro del discurso monológico Kristeva ubica a) el modo representativo de la descripción y la narración épica; b) el discurso histórico; y c) el discurso científico. En los tres, el sujeto asume el papel de dios. En cambio, el discurso dialógico remite a una estructura carnavalesca, antiteológica, antimística $y$ profundamente popular. 
dialógico se encuentra hoy en día ampliamente extendido gracias a las posibilidades comunicativas que brinda Internet.

Finalmente, este estudio evidencia que las comunidades de software libre, entendidas como comunidades de conocimiento que practican el diálogo socrático y la cooperación, se encuentran lejos de la visión lineal y determinista del desarrollo tecnológico promovida por la teoría económica. En primer lugar, porque forman parte de un proceso de desarrollo descentralizado, donde el producto tecnológico no se considera cerrado y terminado, la explotación comercial es secundaria y priva un gran interés por ayudar al prójimo. En segundo lugar, porque los usuarios, en su mayoría estratégicos, tienen un gran control sobre la informática que usan y pueden construir socialmente soluciones o mejoras a sus programas.

\section{CONCLUSIONES}

Las principales conclusiones de este trabajo son las siguientes:

1. La mayoría de los integrantes de las comunidades de software libre y código abierto de Costa Rica suelen ser jóvenes informáticos, predominantemente hombres entre 21 y 32 años, con una escasa participación femenina, el 95\% de la muestra fueron hombres y solo el 5\% mujeres. Estos datos reflejan la poca participación de las mujeres costarricenses, tanto en las comunidades como en los proyectos de desarrollo de software libre y código abierto.

2. Las propuestas comunitarias que surgen en la red se caracterizan por ser efímeras y discontinuas, y las comunidades costarricenses no son la excepción. No siempre estos espacios socio-técnicos consiguen adquirir cierta continuidad en el tiempo que les permita constituirse en referentes relevantes. Además, muchos de los miembros pertenecen a más de una comunidad, dadas las posibilidades comunicativas que ofrece hoy en día Internet. Teniendo en cuenta lo anterior, el Grupo de Usuarios de Linux de Costa Rica (GULCR) ha sido, desde su fundación a finales de los noventa, la comunidad de software libre más activa y numerosa (hay inscritas en la lista de correo más de 300 personas), con incontables mensajes publicados y decenas de festivales de instalación $y$ conferencias realizadas.

3. Independientemente del tiempo que perduren, las comunidades de software libre $y$ open source constituyen espacios de conocimiento. Este conocimiento no es en absoluto abstracto, sino que se refiere a conocimientos técnicos sobre como configurar sistemas GNU/Linux. El apoyo y la ayuda de la comunidad al usuario es siempre muy importante, pues es el único soporte técnico que existe. La comunidad de usuarios desempeña lo que en la empresa es el departamento de "ayuda al cliente". En ese sentido, las comunidades de Costa Rica son generadoras de conocimiento, el cual es compartido libremente a través de los foros y las listas de correo.

4. Las comunidades costarricenses practican un hacktivismo digitalmente correcto, es decir, sus miembros, la mayoría hackers con amplios conocimientos técnicos, suelen privilegiar lo tecnológico sobre lo políticosocial. Son grupos muy técnicos, y puede dar la impresión de ser sectarios y cerrados. Muchas de las comunidades estudiadas se caracterizan por la tecnomeritocracia, es decir, los usuarios son valorados por sus conocimientos y capacidad para resolver problemas, independientemente de sus títulos académicos o puesto laboral. Esta actitud no siempre es comprendida, de ahí que para muchos usuarios tácticos, comunidades como GULCR sean discriminativas y elitistas.

5. Finalmente, las conversaciones de la comunidad son registradas digitalmente $y$ constituyen fuentes de información y de consulta, $y$ al leerlas es evidente que estamos ante una forma de discurso: el discurso dialógico. El modelo del diálogo socrático y los procedimientos retóricos de sincrisis y anacrisis, como estructuras discursivas están presentes en las prácticas de interacción de los grupos en línea, especialmente aquellos como las comunidades de software libre y código abierto que buscan aclarar dudas sobre tecnología de una manera dialógica y participativa. 
REFERENCIAS

Anderson, Benedict. Imagined Communities: Reflections on the Origin and Spread of Nationalism. 1991. Verso.

Castells, Manuel. The Internet Galaxy: Reflections on the Internet, Business, and Society. New York: Oxford University Press, 2001.

Denzin, Norman. The research act. Chicago: Aldine, 1970.

Flichy, Patrice. L' innovation technique. París: La Découverte, 2003.

Flores, Sofía. "Las comunidades de software libre de Costa Rica". [Tesis de Maestría]. San José: Universidad de Costa Rica, 2009 .

Gibson, William. Neuromancer. Ace Books. 2000.

Kelle, Udo. "Sociological explanations between micro and macro and the integration of qualitative and quantitative methods". Forum Qualitative Sozialforschung/ Forum: Qualitative Social Research 2(1).
2001. En: <URLhttp://nbn-resolving.de/ urn:nbn:de:0114-fqs010159>

Kristeva, Julia. Semiótica. Tomo 2. Madrid: Fundamentos, 1981.

Lizama Mendoza, Jorge Alberto. "Hackers en el contexto de la sociedad de la información". [Tesis doctoral]. México: UnAM, Facultad de Ciencias Políticas y Sociales, 2005.

Rheingold, Howard. The Virtual Community: Homesteading on the Electronic Frontier. MIT Press. 2000.

Scolari, Carlos. Hacer clic: Hacia una sociosemiótica de las interacciones digitales. Barcelona: Gedisa, 2004.

Wilbur, Shawn. Archaeology of cyberspace. The cybercultures reader. 2000: 45-55.

ENTREVISTAS

Guerra, Mario. (2007). San José.

Magallón, Marcelo. (2007). San José.

Magaña, Roberto. (2007). San José. 